\title{
Chemotherapy-associated steatohepatitis was concomitant with epicardial adipose tissue volume increasing in breast cancer patients received neoadjuvant chemotherapy
}

\author{
Xiaoxia Wang \\ Chongqing University Cancer Hospital \\ Yuchuan Tan \\ Chongqing University Cancer Hospital \\ Jun Qi \\ Chongqing University Cancer Hospital \\ Daihong Liu \\ Chongqing University Cancer Hospital \\ Hesong Shen \\ Chongqing University Cancer Hospital

\section{Yong Tan} \\ Chongqing University Cancer Hospital \\ Lei Wang \\ Chongqing University Cancer Hospital \\ Yipeng Zhang \\ Chongqing University Cancer Hospital \\ Xin Ma \\ Chongqing University Cancer Hospital \\ Jiazheng Wang
}

MSC Clinical \& Technical Solutions Philips Healthcare

Jiuquan Zhang ( $\sim$ zhangjq_radiol@foxmail.com )

Chongqing University Cancer Hospital https://orcid.org/0000-0003-0239-6988

\section{Research article}

Keywords: Chemotherapy-associated steatohepatitis, Epicardial adipose tissue volume, Breast cancer, Neoadjuvant chemotherapy, Cardiovascular disease

Posted Date: April 9th, 2021

DOI: https://doi.org/10.21203/rs.3.rs-403374/v1 
License: (a) (i) This work is licensed under a Creative Commons Attribution 4.0 International License. Read Full License 


\section{Abstract \\ Background}

The hepatotoxicity and cardiotoxicity caused by neoadjuvant chemotherapy (NAC) are of increasing concern to clinicians in breast cancer (BC) patients. The aim of this study was to investigate the prevalence of chemotherapy-associated steatohepatitis (CASH), quantitate the epicardial adipose tissue (EAT) volume in BC patients, and explore the correlations of these parameters with blood lipid levels in BC patients who received NAC.

\section{Methods}

From October 2018 to April 2020, BC patients were retrospectively reviewed. Clinical and laboratory data were collected. The patients were divided into BC-non-NAC group and BC-NAC group (BC-NAC subgroups $1 \sim 4$ according to the number of NAC cycles). The prevalence of CASH was evaluated using quantitative MRI mDIXON-Quant examinations, from which the proton density fat fraction (PDFF) was assessed. The EAT volume was quantified on chest $\mathrm{CT}$ by semi-automatic volume analysis software. Pearson correlation coefficients were calculated for the associations among PDFF values, EAT volumes and blood lipid levels.

\section{Results}

A total of 662 women (BC-non-NAC group: 445 patients; BC-NAC group: 217 patients) were included. The prevalence of CASH in BC-NAC group was significantly higher than the prevalence of hepatic steatosis in the BC-non-NAC group ( $42.8 \%$ vs. $33.3 \%, p<0.001)$. As the number of NAC cycles increased, the prevalence of CASH increased in the BC-NAC subgroups $(p=0.034)$. EAT volume was measured in 561 of $662 \mathrm{BC}$ patients, and was significantly higher in the BC-NAC group than in the BC-non-NAC group $(137.26 \pm 53.48 \mathrm{ml}$ vs. 125.14 $\pm 58.77 \mathrm{ml}, p=0.020$ ). A moderate positive correlation was observed between the hepatic PDFF value and EAT volume $(r=0.411, p<0.001)$, and these parameters were also positively correlated with triglycerides in the BCNAC group ( $r=0.386, p=0.004 ; r=0.338, p<0.001$, respectively).

\section{Conclusions}

NAC significantly increased the prevalence of CASH and EAT volume and there was significant positive correlation among the hepatic PDFF value, EAT volume and triglycerides, which indicate the abnormal lipid metabolism caused by NAC may be another pathway to induce cardiovascular disease in BC patients.

\section{Background}

Breast cancer (BC) has the highest incidence among cancers affecting women worldwide [1]. Neoadjuvant chemotherapy (NAC) has been established as a standard treatment of care for most BC, due to its ability to downstage, reduce metastasis, detect drug sensitivity, and improve the possibility for breast-conserving 
therapy [2]. With the wide application of NAC, the hepatotoxicity [3] and cardiotoxicity [4, 5] caused by chemotherapeutic drugs have been given more attention by clinicians.

The ability of many chemotherapeutics to cause steatohepatitis has become more evident coining the term chemotherapy-associated steatohepatitis (CASH) [6-8]. CASH is characterized by a typical pathological model of lipid accumulation in hepatocytes, often accompanied by oxidative stress and inflammatory events, involving secondary cell types [7]. Although CASH is considered reversible, it will persist for a long time after chemotherapy [9]. Therefore, it is important to identify CASH and reinforce monitoring during treatment $[8,10]$. Core biopsy combined with semiquantitative histologic grading is the gold reference for determining hepatic fat content [11]. However, invasiveness, sampling error, observer dependence and spatial heterogeneity [12] have weakened the role of liver biopsy in monitoring and clinical research. A noninvasive magnetic resonance imaging (MRI) mDIXON-Quant sequence is capable of measuring the proton density fat fraction (PDFF) [13], which provides a measure of tissue triglyceride concentration and is increasingly accepted as an imaging biomarker to quantify liver fat content [14].

BC patients who receive cardiotoxic chemotherapy agents (such as anthracycline, cyclophosphamide and trastuzumab etc) have a significantly increased incidence of cardiovascular disease (CVD) [15-17]. Early identification of the appearance of cardiovascular alterations in BC patients during NAC could help tailor cancer treatment through the adoption of preventive or intervention measures [18]. Alterations in epicardial adipose tissue (EAT) volume are associated with coronary artery plaque development and myocardial ischemia, increased coronary calcium and an increased incidence of cardiovascular adverse events [19]. It turned out to provide incremental prognostic value over coronary artery calcium scoring [20]. Owing to its high spatial resolution and true volume coverage of the entire heart, CT is currently the preferred method to determine EAT volume [21]. To our knowledge, in BC patients, the pattern of changes in EAT volume after received NAC has not been reported.

CASH is a special type of nonalcoholic fatty liver disease (NAFLD). NAFLD is one of the major driving forces for CVD and is recognized as an independent risk factor, that confers significant morbidity and mortality in this population [22, 23]. Clinical and epidemiological studies [24-26] have confirmed the role of NAFLD in the development of different CVD manifestations, such as atherosclerotic CVD, left ventricular dysfunction, cardiac conduction system abnormalities and ischemic stroke. However, it is not well understood in BC patients received NAC, if CASH leads to a high risk for cardiovascular events or whether cardiovascular risk varies across the spectrum of CASH.

Therefore, the first endpoint of our study was to quantify liver fat content by MRI mDIXON-Quant examinations to identify the prevalence of $\mathrm{CASH}$, and measure the EAT volume on chest $\mathrm{CT}$ by semi-automatic volume analysis software to explore the pattern of changes in BCpatients after received NAC. The second endpoint was to investigate the correlations among the hepatic PDFF value, EAT volume and blood lipid levels.

\section{Methods}

\section{Study Population}


This study was approved by the ethics committee of the hospital (No.: CZLS20200215-A). The requirement for written informed consent was waived for this retrospective study. Clinical records were reviewed for 863 patients who had confirmed breast lesions and underwent MRI mDIXON-Quant examinations of the liver between October 2018 and April 2020. Patients were included if malignant breast lesions were histologically confirmed. According to whether they had received NAC treatment, the patients were divided into BC-non-NAC group and BC-NAC group. Next, patients were excluded if other potential causes of chronic liver disease were identified, including hepatitis B/C virus, liver metastases, hypothyroidism, alcohol use (>30 g/week), and other malignant tumors. For the BC-NAC group, patients who were receiving antineoplastic therapy other than NAC therapy were further excluded. Finally, 662 BC patients (445 patients in the BC-non-NAC group and 217 patients in the BC-NAC group) were included for MRI-PDFF measurement and analysis.

Then, among the 662 BC patients, we screened out patients who simultaneously had undergone chest CT within one week with liver MRI examinations. Patients were excluded if the software failed to detect the epicardial contour. Finally, 561 BC patients (383 patients in the BC-non-NAC group and 178 patients in the BCNAC group) were included for CT-EAT volume measurement and analysis. The details are demonstrated in Fig. 1.

\section{Clinical and Laboratory Data}

The clinical data included age, body mass index (BMI), menstruation state, and diagnosis of hypertension and type 2 diabetes mellitus. The histopathological diagnosis data obtained before NAC treatment were retrieved from the hospital's electronic medical records. The laboratory data included alanine aminotransferase (U/L), aspartate aminotransferase $(\mathrm{U} / \mathrm{L})$, direct bilirubin $(\mu \mathrm{mol} / \mathrm{L})$, indirect bilirubin $(\mu \mathrm{mol} / \mathrm{L})$, total protein $(\mathrm{g} / \mathrm{L})$, albumin $(\mathrm{g} / \mathrm{L})$, creatinine $(\mu \mathrm{mol} / \mathrm{L})$, urea $(\mathrm{mmol} / \mathrm{L})$, serum iron $(\mu \mathrm{mol} / \mathrm{L})$, cholesterol $(\mathrm{mmol} / \mathrm{L})$, triglycerides $(\mathrm{mmol} / \mathrm{L})$, high-density lipoprotein ( $\mathrm{mmol} / \mathrm{L})$, and low-density lipoprotein ( $\mathrm{mmol} / \mathrm{L})$. Blood samples were collected from all subjects before $10 \mathrm{AM}$ after a $12 \mathrm{~h}$ overnight fast. All laboratory tests were performed on the same day using standard laboratory methods before treatment.

\section{NAC Protocol}

The treatment protocol followed the National Comprehensive Cancer Network guidelines [27]. All patients received 4 cycles, 6 cycles, or 8 cycles of NAC prior to breast surgery. The NAC regimens were taxane-based, anthracycline-based, or anthracycline and taxane-based. We recorded the number of NAC cycles concomitant with liver MRI examination. Based on the number of cycles of NAC, the BC-NAC group patients were divided into four subgroups: subgroup 1 (1 2 cycles), subgroup 2 (3 4 cycles), subgroup 3 (5 6 cycles) and subgroup 4 (7 8 cycles).

\section{Liver MRI mDIXON-Quant Sequence Protocol}

All patients underwent liver mDIXON-Quant MRI in a 3.0 T scanner (Ingenia 3.0T, Philips Healthcare, Best, Netherlands) with a 16-channel breast coil, a 3-dimensional fast-field-echo sequence with multiple echoes was used for the signal acquisition, and the water, fat, $2^{*}$, and fat fraction images were generated in real-time on the MR scanner console. The scanning parameters were as follows: TR, $5.6 \mathrm{~ms} ; 6$ echoes with the first TE of $0.97 \mathrm{~ms}$ and a TE step size of $0.7 \mathrm{~ms}$; FOV, $400 \times 350 \times 210 \mathrm{~mm}^{3}$; FA, $3^{\circ}$; resolution, $2.5 \times 2.5 \times 3.0 \mathrm{~mm}^{3}$; SENSE, AP, 2, FH, 1; NSA, 1; and scan time, 14 s. 


\section{Liver Fat Quantification}

The hepatic PDFF values were respectively measured and recorded from six regions of interest (ROIs) in two transverse sections through the first hepatic portal vein and below the second portal vein in the fat fraction image (Fig. 2). All ROIs were distributed in the hepatic parenchyma, and the biliary, vascular, and extrahepatic structures were excluded. The area of each ROI was approximately $300 \mathrm{~mm}^{2}$. On the basis of previous study [28], the following threshold values for PDFF were used for diagnosis: < 5.0\%, grade 0 (no fat content); 5.0\% $14.0 \%$, grade 1 (mild fat content); $14.0 \%$ 28.0\%, grade 2 (moderate fat content); and > 28.0\%, grade 3 (high fat content).

\section{Chest CT Image Acquisition Protocol}

Image data were acquired on a 2.5 generation dual-source CT unit (SOMATOM Drive, Siemens Healthineers, Forchheim, Germany). Non-contrast CT images for analysis of EAT volume were acquired ( $90 \mathrm{kV}$ tube voltage, $185 \mathrm{mAs}$ tube current). Automatic exposure control (CARE Dose 4D, Siemens Healthineers) was used in all scans. The settings for the scanners were as follows: collimation, $128 \times 0.6 \mathrm{~mm}$; rotation time, $0.5 \mathrm{~s}$; reformatted section thickness, $1.5 \mathrm{~mm}$; reformatted section increment, $1.5 \mathrm{~mm}$.

\section{Analysis of EAT Volume}

The EAT volume was measured on non-contrast chest CT images by using dedicated semi-automatic volume analysis software (Cardiac Risk Assessment Prototype, Siemens Healthineers, Forchheim, Germany). The reader was blinded to the patient characteristics and clinical data. All data sets were checked for coverage of the entire epicardial sac. First, the upper and lower limits of the pericardial sac were identified by the reader defined as the bifurcation of the pulmonary trunk and, respectively, the slice caudal to the posterior descending artery. Second, the contour of the pericardial sac was automatically traced and adjusted by the reader, if necessary (Fig. 3). Finally, the EAT volume $(\mathrm{ml})$ was automatically calculated by the inclusion of contiguous 3D voxels with CT attenuations between - 190 to $-30 \mathrm{HU}$ in the volume of interest as this is a threshold commonly used for EAT volume analysis in non-contrast scans [29].

To ensure the PDFF and EAT reproducibility, we randomly selected 30 patients to assess interobserver agreement (Y.C.T., with 11 years of experience, and L.W., with 6 years of experience) and 30 patients to assess intraobserver agreement (Y.C.T), respectively. The 6 ROIs of PDFF measurements and EAT analysis were repeated twice, with an interval of at least 1 month, following the same procedure. Intraclass correlation coefficients (ICCS) were utilized to evaluate inter- and intraobserver agreement of the PDFF value and EAT volume.

\section{Statistical analysis}

Statistical analyses were performed using commercially available statistical software (SPSS software, version 25.0; Armonk, US). Descriptive statistics are summarized as the mean \pm standard deviation. Categorical variables are expressed as numbers (percentages) and were calculated by the $\chi^{2}$ test or Fisher's exact test. For binary analysis, parametric data was compared using $t$-test, non-parametric data were compared using the Mann-Whitney $U$ test for continuous variables between the BC-non-NAC group and BC-NAC group. One-way ANOVA with Tukey's post hoc test (normally distributed data) and the non-parametric Kruskal-Wallis $H$ test 
(non-normally distributed data) were performed among BC-NAC subgroups. Correlations among laboratory data, number of cycles of NAC, PDFF value and EAT volume were assessed using Pearson correlation. The level of significance was defined as $p<0.05$.

\section{Results}

\section{Clinical and Laboratory Data Characteristics}

Finally, $662 \mathrm{BC}$ patients were included for MRI-PDFF analysis in the study: 445 patients (mean age 51.89 years, range: $26 \sim 82$ years) in the BC-non-NAC group and 217 patients in the BC-NAC group (mean age of 50.78 years, range: $21 \sim 71$ years). In addition, $561 \mathrm{BC}$ patients who had simultaneous chest CT scans were included for CT-EAT volume analysis, 383 patients in the BC-non-NAC group and 178 patients in the BC-NAC group.

The demographics, comorbidities, lesion type and serum laboratory values for $662 \mathrm{BC}$ patients are summarized in Table 1. There were no significant differences in age, BMI, diagnosis of hypertension and diabetes mellitus or $\mathrm{BC}$ pathological type between the two groups. The alanine and aspartate aminotransferase levels were higher in the BC-NAC group than in the BC-non-NAC group (both $p<0.001$ ). The levels of indirect bilirubin, total protein, serum iron (all $p<0.001)$, albumin $(p=0.001)$ and high-density lipoprotein $(p=0.018)$ were lower in the BC-NAC group than in the BC-non-NAC group. 
Table 1

Demographics, comorbidities, lesion type and serum laboratory values for 662 breast cancer patients stratified by NAC treatment

\begin{tabular}{|c|c|c|c|}
\hline Variable & $\begin{array}{l}\text { BC-non-NAC group } \\
(n=445)\end{array}$ & $\begin{array}{l}\text { BC-NAC group } \\
(n=217)\end{array}$ & $p$ value \\
\hline \multicolumn{4}{|l|}{ Demographics } \\
\hline Age, Mean $\pm S D$, years & $51.89 \pm 10.12$ & $50.78 \pm 9.3$ & 0.177 \\
\hline Body mass index $\left(\mathrm{BMI}, \mathrm{kg} / \mathrm{m}^{2}\right)$ & $24.48 \pm 3.27$ & $24.32 \pm 3.33$ & 0.524 \\
\hline \multicolumn{4}{|l|}{ Menstruation State } \\
\hline Premenopausal & $235(52.8 \%)$ & $134(61.8 \%)$ & 0.030 \\
\hline Postmenopausal & $210(47.2 \%)$ & $83(38.2 \%)$ & \\
\hline Hypertension, n (\%) & $60(13.5 \%)$ & $25(11.5 \%)$ & 0.479 \\
\hline Diabetes mellitus, n (\%) & $30(6.7 \%)$ & $15(6.9 \%)$ & 0.935 \\
\hline \multicolumn{4}{|l|}{ Breast lesion type } \\
\hline Invasive ductal/lobular carcinoma & 417 & 209 & 0.543 \\
\hline DCIS with microinvasion & 18 & 5 & \\
\hline Medullary carcinoma & 3 & 0 & \\
\hline Mucinous carcinoma & 5 & 3 & \\
\hline Phyllodes tumor & 2 & 0 & \\
\hline Serum laboratory values & $(n=435)$ & $(n=212)$ & \\
\hline Alanine aminotransferase (U/L) & $20.26 \pm 18.19$ & $31.03 \pm 24.56$ & $<0.001$ \\
\hline Aspartate aminotransferase (U/L) & $22.55 \pm 15.25$ & $29.01 \pm 17.78$ & $<0.001$ \\
\hline Creatinine $(\mu \mathrm{mol} / \mathrm{L})$ & $51.66 \pm 9.81$ & $51.55 \pm 12.20$ & 0.903 \\
\hline Urea (mmol/L) & $5.11 \pm 1.54$ & $4.94 \pm 1.41$ & 0.185 \\
\hline Direct bilirubin ( $\mu \mathrm{mol} / \mathrm{L})$ & $3.41 \pm 1.46$ & $3.09 \pm 4.03$ & 0.142 \\
\hline Indirect bilirubin $(\mu \mathrm{mol} / \mathrm{L})$ & $8.51 \pm 4.00$ & $6.69 \pm 3.52$ & $<0.001$ \\
\hline Total protein $(\mathrm{g} / \mathrm{L})$ & $74.22 \pm 6.49$ & $70.30 \pm 8.15$ & $<0.001$ \\
\hline Albumin (g/L) & $42.71 \pm 3.58$ & $41.45 \pm 5.52$ & 0.001 \\
\hline Serum iron $(\mu \mathrm{mol} / \mathrm{L})$ & $15.16 \pm 6.10$ & $12.67 \pm 4.98$ & $<0.001$ \\
\hline Blood lipid & $(n=199)$ & $(n=53)$ & \\
\hline Cholesterol (mmol/L) & $4.83 \pm 1.10$ & $4.59 \pm 0.90$ & 0.162 \\
\hline
\end{tabular}




\begin{tabular}{|llll|}
\hline Variable & $\begin{array}{l}\text { BC-non-NAC group } \\
(\mathbf{n}=\mathbf{4 4 5})\end{array}$ & $\begin{array}{l}\text { BC-NAC group } \\
(\mathbf{n}=\mathbf{2 1 7})\end{array}$ & $\boldsymbol{p}$ value \\
\hline Triglycerides (mmol/L) & $1.51 \pm 1.73$ & $1.36 \pm 0.61$ & 0.523 \\
\hline High-density lipoprotein (mmol/L) & $1.56 \pm 0.34$ & $1.44 \pm 0.26$ & 0.018 \\
\hline Low-density lipoprotein (mmol/L) & $2.67 \pm 0.79$ & $2.58 \pm 0.70$ & 0.480 \\
\hline Note.-NAC, neoadjuvant chemotherapy; DCIS, ductal carcinoma in situ. & \\
\hline
\end{tabular}

\section{MRI-PDFF Analysis}

The hepatic PDFF values of the 662 patients in the BC-NAC group and BC-non-NAC group are summarized in Table 2. According to the PDFF values, hepatic steatosis was diagnosed in $33.3 \%$ (148 of 445 patients) of patients in the BC-non-NAC group [mild, 31.0\% (138 patients); moderate, 2.3\% (10 patients)], and 42.8\% (93 of 217 patients) of patients in the BC-NAC group [mild, 30.9\% (67 patients); moderate, $11.5 \%$ ( 25 patients); severe, $0.4 \%$ ( 1 patient)]. There were significant differences in the prevalence of hepatic steatosis between the two groups $(p<0.001)$. In the quantitative analysis, the PDFF value in the BC-NAC group was significantly higher than those in the BC-non-NAC group $(6.30 \pm 5.16 \%$ vs. $5.26 \pm 4.67 \%, p=0.009)$. 
Table 2

The measurement of hepatic PDFF value and EAT volume for breast cancer patients stratified by cycles of NAC

\begin{tabular}{|c|c|c|c|c|c|c|c|c|}
\hline \multirow[t]{2}{*}{ Variable } & \multirow{2}{*}{$\begin{array}{l}\text { Non- } \\
\text { NAC } \\
\text { group }\end{array}$} & \multirow{2}{*}{$\begin{array}{l}\text { NAC } \\
\text { group }\end{array}$} & \multirow{2}{*}{$\begin{array}{l}p \\
\text { value }\end{array}$} & \multicolumn{4}{|c|}{ NAC subgroups } & \multirow{2}{*}{$\begin{array}{l}p \\
\text { value }\end{array}$} \\
\hline & & & & Subgroup & $\begin{array}{l}\text { Subgroup } \\
2\end{array}$ & $\begin{array}{l}\text { Subgroup } \\
3\end{array}$ & $\begin{array}{l}\text { Subgroup } \\
4\end{array}$ & \\
\hline $\begin{array}{l}\text { No. of } \\
\text { patients with } \\
\text { liver PDFF } \\
\text { analysis }\end{array}$ & 445 & 217 & & 40 & 71 & 57 & 49 & \\
\hline \multicolumn{9}{|l|}{$\begin{array}{l}\text { PDFF } \\
\text { classification }\end{array}$} \\
\hline$<5 \%$ & $\begin{array}{l}297 \\
(66.7 \%)\end{array}$ & $\begin{array}{l}124 \\
(57.2 \%)\end{array}$ & $\begin{array}{l}< \\
0.001\end{array}$ & $\begin{array}{l}31 \\
(77.5 \%)\end{array}$ & $\begin{array}{l}35 \\
(61.4 \%)\end{array}$ & $\begin{array}{l}38 \\
(53.5 \%)\end{array}$ & $\begin{array}{l}20 \\
(40.8 \%)\end{array}$ & 0.034 \\
\hline $5 \%-14 \%$ & $\begin{array}{l}138 \\
(31.0 \%)\end{array}$ & $\begin{array}{l}67 \\
(30.9 \%)\end{array}$ & & $6(15.0 \%)$ & $10(17.5)$ & $\begin{array}{l}18 \\
(25.4 \%)\end{array}$ & $\begin{array}{l}15 \\
(30.6 \%)\end{array}$ & \\
\hline $14 \%-28 \%$ & $\begin{array}{l}10 \\
(2.3 \%)\end{array}$ & $\begin{array}{l}25 \\
(11.5 \%)\end{array}$ & & $3(7.5 \%)$ & $\begin{array}{l}12 \\
(21.1 \%)\end{array}$ & $\begin{array}{l}15 \\
(21.1 \%)\end{array}$ & $\begin{array}{l}13 \\
(26.5 \%)\end{array}$ & \\
\hline$>28 \%$ & $0(0 \%)$ & $\begin{array}{l}1 \\
(0.4 \%)\end{array}$ & & $0(0 \%)$ & $0(0 \%)$ & $0(0 \%)$ & $1(2.1 \%)$ & \\
\hline \multicolumn{9}{|l|}{ PDFF (\%) } \\
\hline $\begin{array}{l}\text { Left liver } \\
\text { lobe }\end{array}$ & $\begin{array}{l}4.67 \pm \\
4.53\end{array}$ & $\begin{array}{l}5.74 \pm \\
5.53\end{array}$ & 0.008 & $\begin{array}{l}4.62 \pm \\
3.65\end{array}$ & $\begin{array}{l}4.92 \pm \\
3.44\end{array}$ & $\begin{array}{l}6.31 \pm \\
7.14\end{array}$ & $\begin{array}{l}7.47 \pm \\
6.63\end{array}$ & 0.035 \\
\hline $\begin{array}{l}\text { Right liver } \\
\text { lobe }\end{array}$ & $\begin{array}{l}5.55 \pm \\
4.83\end{array}$ & $\begin{array}{l}6.58 \pm \\
5.24\end{array}$ & 0.013 & $\begin{array}{l}5.56 \pm \\
3.42\end{array}$ & $\begin{array}{l}5.65 \pm \\
3.37\end{array}$ & $\begin{array}{l}6.63 \pm \\
5.91\end{array}$ & $\begin{array}{l}9.07 \pm \\
6.88\end{array}$ & 0.030 \\
\hline Average & $\begin{array}{l}5.26 \pm \\
4.67\end{array}$ & $\begin{array}{l}6.30 \pm \\
5.16\end{array}$ & 0.009 & $\begin{array}{l}5.33 \pm \\
3.42\end{array}$ & $\begin{array}{l}5.41 \pm \\
3.31\end{array}$ & $\begin{array}{l}6.52 \pm \\
5.89\end{array}$ & $\begin{array}{l}8.54 \pm \\
6.75\end{array}$ & 0.035 \\
\hline $\begin{array}{l}\text { No. of } \\
\text { patients with } \\
\text { EAT volume } \\
\text { analysis }\end{array}$ & 383 & 178 & & 36 & 61 & 40 & 41 & \\
\hline $\begin{array}{l}\text { EAT volume } \\
(\mathrm{ml})\end{array}$ & $\begin{array}{l}125.14 \\
\pm 58.77\end{array}$ & $\begin{array}{l}137.26 \\
\pm 53.48\end{array}$ & 0.020 & $\begin{array}{l}130.20 \pm \\
63.62\end{array}$ & $\begin{array}{l}137.74 \pm \\
50.92\end{array}$ & $\begin{array}{l}141.43 \pm \\
63.73\end{array}$ & $\begin{array}{l}155.08 \pm \\
50.49\end{array}$ & 0.004 \\
\hline
\end{tabular}

The detailed NAC regimens in each group are shown in Table 3. In the BC-NAC subgroup analysis, as the number of NAC cycles increased, the prevalence of hepatic steatosis increased in the 4 subgroups (22.5\% vs. $38.6 \%$ vs. $46.5 \%$ vs. $59.2 \%, p=0.034)$. The PDFF value in subgroup 4 was significantly higher than those in subgroup 1 ( $8.54 \pm 6.75 \%$ vs. $5.33 \pm 3.42 \%$, adjusted $p=0.023$ ) (Fig. 4A). There were no significant differences among subgroups 1, 2 and 3. Moreover, the PDFF value in the right lobe of the liver was significantly higher than those in the left lobe, in the BC-non-NAC group, BC-NAC group and all NAC subgroups (all $p<0.001)$. 
Table 3

Details of the NAC regimens in each subgroup

\begin{tabular}{|llllll|}
\hline & $\begin{array}{l}\text { Subgroup } \mathbf{1} \\
(\mathbf{n = 4 0 )}\end{array}$ & $\begin{array}{l}\text { Subgroup 2 } \\
(\mathbf{n = 5 7 )}\end{array}$ & $\begin{array}{l}\text { Subgroup 3 } \\
(\mathbf{n = 7 1 )}\end{array}$ & $\begin{array}{l}\text { Subgroup 4 } \\
(\mathbf{n}=\mathbf{4 9})\end{array}$ & $\begin{array}{l}\text { Total } \\
(\mathbf{n}=\mathbf{2 1 7})\end{array}$ \\
\hline Taxane-based & 19 & 27 & 34 & 16 & 96 \\
\hline Anthracycline-based & 9 & 14 & 20 & 8 & 51 \\
\hline Anthracycline-and Taxane-based & 12 & 16 & 17 & 25 & 70 \\
\hline Note.-NAC, neoadjuvant chemotherapy. & & & & \\
\hline
\end{tabular}

\section{CT-EAT Volume Analysis}

The EAT volumes of the 561 patients in the BC-NAC group and BC-non-NAC group are also summarized in Table 2. The EAT volume in the BC-NAC group was significantly higher than that in the BC-non-NAC group $(137.26 \pm 53.48 \mathrm{ml}$ vs. $125.14 \pm 58.77 \mathrm{ml}, p=0.020)$. In the BC-NAC subgroup analysis, as the number of NAC cycles increased, the EAT volume also increased in the 4 subgroups. The EAT volume in subgroup 4 was significantly higher than those in subgroup $1(155.08 \pm 50.49 \mathrm{ml}$ vs. $130.20 \pm 63.62 \mathrm{ml}$, adjusted $p=0.002)$ (Fig. 4B). There were no significant differences among subgroups 1, 2 and 3.

\section{Correlation Analysis}

The Pearson correlation coefficients among laboratory data, cycles of NAC, PDFF value and EAT volume in the BC-NAC group are shown in Table 4. Overall, the PDFF value was positively correlated with alanine aminotransferase, aspartate aminotransferase, total protein, and triglycerides $(r=0.211 \sim 0.386 ; p<0.001 \sim$ $0.005)$ and was negatively correlated with high-density lipoprotein $(r=-0.328, p=0.017)$. The number of cycles of NAC had a positive correlation with the hepatic PDFF value $(r=0.252, p<0.001)$ and EAT volume $(r=0.259$, $p<0.001)$. The EAT volume was positively correlated with triglycerides $(r=0.338, p<0.001)$. A moderate positive correlation was observed between the hepatic PDFF value and EAT volume $(r=0.411, p<0.001)$ (Fig. 5). 
Table 4

Correlation analysis in the NAC group

\begin{tabular}{|lcc|}
\hline & Correlation coefficient $(\boldsymbol{\eta})$ & $p$ value \\
\hline PDFF (\%) & & \\
\hline Alanine aminotransferase (U/L) & 0.351 & $<0.001$ \\
\hline Aspartate aminotransferase (U/L) & 0.211 & 0.002 \\
\hline Total protein (g/L) & 0.193 & 0.005 \\
\hline Triglycerides (mmol/L) & 0.386 & 0.004 \\
\hline High-density lipoprotein (mmol/L) & -0.328 & 0.017 \\
\hline Cycles of NAC & & $<0.001$ \\
\hline PDFF (\%) & 0.252 & $<0.001$ \\
\hline EAT volume (ml) & 0.259 & \\
\hline EAT volume (ml) & & $<0.001$ \\
\hline Triglycerides (mmol/L) & 0.338 & $<0.001$ \\
\hline PDFF (\%) and EAT volume (ml) & 0.411 & \\
\hline Note.PDFF, proton density fat fraction; EAT, epicardial adipose tissue; NAC, neoadjuvant chemotherapy. \\
\hline
\end{tabular}

\section{Interobserver and Intraobserver Agreement}

The inter- and intraobserver agreements were excellent for all measurements, with ICC values greater than 0.80. The interobserver ICC values for PDFF and EAT were 0.974 and 0.912 , respectively, and the intraobserver values were 0.986 and 0.945 , respectively. Bland-Altman analysis showed that the PDFF value and EAT volume showed both good inter- and intraobserver agreement. Interobserver: PDFF, 169/180 (93.8\%), Fig. 6A; EAT volume, 30/30 (100\%), Fig. 6B; Intraobserver: PDFF, 173/180 (96.1\%), Fig. 6C; EAT volume, 28/30 (93.3\%), Fig. 6D.

\section{Discussion}

In BC patients, hepatotoxicity and cardiovascular disease caused by NAC treatment are of increasing concern to clinicians. $\mathrm{CASH}$ is a very common manifestation of hepatotoxicity, but it has not received much clinical attention. In our study, we quantitatively assessed liver PDFF to determine the prevalence of CASH and found that the prevalence in $\mathrm{BC}$ patients who received NAC was as high as $42.8 \%$. Furthermore, we conducted quantitative measurement of EAT volume and found a significant increase in EAT volume in BC patients who received NAC, indicating an increased risk for CVD in these patients. The prevalence of CASH and EAT volume both increased as the number of NAC cycles increased, and there was significant positive correlation among the hepatic PDFF value, EAT volume and triglycerides. Our results indicate that the abnormal lipid metabolism 
caused by NAC may be another pathway to inducing CVD in BC patients, which should give more attention by clinicians.

A strong association between PDFF and histopathological steatohepatitis has been identified in previous studies [30,31], suggesting that MRI is a reliable tool for CASH evaluation. In our study, the prevalence of $\mathrm{CASH}$ was as high as $42.8 \%$ in the BC patients who received NAC, consistent with previous study [32]. CASH is a consequence of mitochondrial function alterations [8]. The $\beta$-oxidation of fatty acids takes place in the mitochondria and in peroxisomes [7]. Some treatments can induce steatosis by decreasing fatty acid $\beta$ oxidation, thus generating oxidative stress via the generation of reactive oxygen species and accumulation in hepatocytes [33]. A previous study [34] found that there was a preferential storage of lipids in the right liver lobe in the presence of excessive accumulation of triglycerides, which was evidenced in our study. This result could be explained by the streamline theory of the portal vein [35].

According to statistics, the mortality rate among tumor patients who develop some cardiovascular event is high, with values more than $60 \%$ at the time of assessment within 2 years [36]. EAT spreads between the myocardium and visceral pericardium and is a highly metabolic and inflammatory active visceral adipose tissue [37]. Evidence has emerged that metabolic processes within the EAT influence atherosclerotic plaque formation by inducing endothelial dysfunction, inflammatory responses and smooth muscle cell proliferation by endocrine and paracrine mechanisms of secreted pro-inflammatory cytokines and adipokines [38]. Based on fully automated EAT volume and attenuation quantification analysis can provide prognostic value for asymptomatic patients [20]. As a result of these non-invasive imaging procedures, EAT measurements are increasingly being performed in the general population, including patients with CVD, obesity, and diabetes [39]. In our study, we measured the EAT volume in BC patients and found EAT volume increase in BC patients who received NAC. It may indicate that the EAT volume increase is related to the cardiotoxicity caused by NAC. To our knowledge, there has been no report on the change of EAT volume in BC patients who received NAC.

Current evidence has shown that patients with NAFLD are at high risk for CVD, which is the main cause of death in these subjects [40], and there is a strong relation between the two conditions. Chemotherapeutic agents can lead to mitochondrial function alterations, resulting in an increase in the level of serum free fatty acids, which will lead to cellulite enlargement, visceral fat deposition and ectopic fat deposition when it exceeds the storage capacity of adipose tissue [41]. In our study, it was observed that there was a moderate positive correlated between the grading of CASH and EAT volume, and these parameters were also correlation with triglycerides. Therefore, we speculate that there may be a certain correlation between the prevalence of CASH and CVD, and the abnormal lipid metabolism caused by NAC in BC patients may be another pathway to inducing CVD.

Chemotherapy-associated liver injuries are associated with higher morbidity and mortality [9]. Therefore, liver function needs to be monitored throughout NAC courses. For instance, discontinuation of treatment should be considered in the case of transaminase increases [42]. The transaminases in our cohort were increased, while the direct and indirect bilirubin, total protein, albumin and serum iron decreased in $\mathrm{BC}$ patients who received NAC, which was consistent with the results of previous studies $[8,10]$. 
Our study had some limitations. First, our study did not include any reference standard, such as liver biopsy (all patients had only mild liver damage, making the invasive procedure unnecessary) or histopathology to confirm absolute EAT volumes. Second, in our study, the patients in the NAC group were not dynamically monitored. Finally, we did not compare the MRI-PDFF and CT-EAT volume with the values obtained with other imaging methods, such as ultrasound, and these correlation studies may be performed in future studies.

\section{Conclusions}

In conclusion, our study found that the prevalence of CASH was $42.8 \%$ and EAT volume was significantly increased in BC patients who received NAC. There were a significant correlation among the hepatic PDFF value, EAT volume and triglycerides. Our results indicate that the abnormal lipid metabolism caused by NAC may be another pathway to inducing CVD in BC patients.

\section{Abbreviations}

$\mathrm{BC}=$ breast cancer, $\mathrm{BMI}=$ body mass index, $\mathrm{CASH}=$ chemotherapy-associated steatohepatitis, CVD = cardiovascular disease, EAT = epicardial adipose tissue, ICC = intraclass correlation coefficient, MRI = magnetic resonance imaging, NAC = neoadjuvant chemotherapy, PDFF = proton density fat fraction, ROI = region of interest

\section{Declarations}

\section{Ethics approval and consent to participate}

This study was approved by Chongqing University Cancer Hospital institutional review board (No.: CZLS20200215-A).

\section{Consent for publication}

Not applicable.

\section{Availability of data and materials}

The datasets used and/or analyzed during the current study are available from the corresponding author on reasonable request.

\section{Competing interests}

The authors declare that they have no competing interests.

\section{Funding}

This study has received funding by the National Natural Science Foundation of China (Grant No. 82071883), the combination projects of medicine and engineering of the Fundamental Research Funds for the Central Universities in 2019 (Project No. 2019CDYGYB008), the Chongqing key medical research project of 
combination of science and medicine (Grant No. 2019ZDXM007), and the 2019 SKY Imaging Research Fund of the Chinese International Medical Foundation (Project No. Z-2014-07-1912-10).

\section{Authors' contributions}

X.W. and Y.T. contributed to the conception and design of the study, data analysis and writing of the manuscript. J.Q., J.W. and D.L. contributed to performing the experiments and writing and revising the manuscript. Y.T. and L.W. contributed to the data collection. Y.Z., H.S. and X.M. contributed to the data analysis and interpretation of the data. J.Z. is the guarantor of this study and approved the version to be submitted. All authors accept responsibility for the integrity of the data and the accuracy of the data analysis.

\section{Acknowledgements}

The authors thank all volunteers who participated in the study and the staff of the Department of Radiology, Chongqing University Cancer Hospital \& Chongqing Cancer Institute \& Chongqing Cancer Hospital in Chongqing, China, for their selfless and valuable assistance.

\section{Disclosures of Conflicts of Interest:}

The authors of this manuscript declare no relationships with any companies, whose products or services may be related to the subject matter of the article. Jiazheng Wang is an employee of Philips Healthcare.

\section{References}

1. RL S, KD M. A J: Cancer statistics, 2020. Cancer J Clin. 2020;70(1):7-30.

2. Derks $M$, van de Velde $C$. Neoadjuvant chemotherapy in breast cancer: more than just downsizing. The Lancet Oncology. 2018;19(1):2-3.

3. WJ G, BO A, SL RB, WB BHJBACADE, SH FAF. G et al: Breast Cancer, Version 4.2017, NCCN Clinical Practice Guidelines in Oncology. Journal of the National Comprehensive Cancer Network: JNCCN. 2018;16(3):310-20.

4. Banke A, Fosbøl E, Ewertz M, Videbæk L, Dahl J, Poulsen M, Cold S, Jensen M, Gislason G, Schou M, et al. Long-Term Risk of Heart Failure in Breast Cancer Patients After Adjuvant Chemotherapy With or Without Trastuzumab. JACC Heart failure. 2019;7(3):217-24.

5. Matos E, Jug B, Blagus R, Zakotnik B. A Prospective Cohort Study on Cardiotoxicity of Adjuvant Trastuzumab Therapy in Breast Cancer Patients. Arquivos brasileiros de cardiologia. 2016;107(1):40-7.

6. Schumacher J, Guo G. Mechanistic review of drug-induced steatohepatitis. Toxicol Appl Pharmcol. 2015;289(1):40-7.

7. Dash A, Figler R, Sanyal A, Wamhoff B. Drug-induced steatohepatitis. Expert Opin Drug Metab Toxicol. 2017;13(2):193-204.

8. Meunier L, Larrey D. Chemotherapy-associated steatohepatitis. Annals of hepatology 2020.

9. Vigano L, De Rosa G, Toso C, Andres A, Ferrero A, Roth A, Sperti E, Majno P, Rubbia-Brandt L. Reversibility of chemotherapy-related liver injury. Journal of hepatology. 2017;67(1):84-91. 
10. Inci F, Karatas F. Paclitaxel-induced hepatic steatosis in patients with breast cancer. Journal of BUON: official journal of the Balkan Union of Oncology. 2019;24(6):2355-60.

11. M F-R LC. R L: Noninvasive Assessment of Liver Disease in Patients With Nonalcoholic Fatty Liver Disease. Gastroenterology. 2019;156(5):1264-81.e1264.

12. SCN H, HK S, DFY C, SKH W, DKW Y, EKW N. WCW C: Validation of water-fat MRI and proton MRS in assessment of hepatic fat and the heterogeneous distribution of hepatic fat and iron in subjects with nonalcoholic fatty liver disease. Eur J Radiol. 2018;107:7-13.

13. Yokoo T, Shiehmorteza M, Hamilton G, Wolfson T, Schroeder M, Middleton M, Bydder M, Gamst A, Kono Y, Kuo A, et al. Estimation of hepatic proton-density fat fraction by using MR imaging at 3.0 T. Radiology. 2011;258(3):749-59.

14. Meisamy S, Hines C, Hamilton G, Sirlin C, McKenzie C, Yu H, Brittain J, Reeder S. Quantification of hepatic steatosis with T1-independent, T2-corrected MR imaging with spectral modeling of fat: blinded comparison with MR spectroscopy. Radiology. 2011;258(3):767-75.

15. Chaosuwannakit N, D'Agostino R, Hamilton C, Lane K, Ntim W, Lawrence J, Melin S, Ellis L, Torti F, Little W, et al. Aortic stiffness increases upon receipt of anthracycline chemotherapy. Journal of clinical oncology: official journal of the American Society of Clinical Oncology. 2010;28(1):166-72.

16. Koelwyn G, Lewis N, Ellard S, Jones L, Gelinas J, Rolf J, Melzer B, Thomas S, Douglas P, Khouri M, et al. Ventricular-Arterial Coupling in Breast Cancer Patients After Treatment With Anthracycline-Containing Adjuvant Chemotherapy. Oncologist. 2016;21(2):141-9.

17. Zhang W, Xie K, Fu S, Jiang H, Fang M, Lian Y, Geng W, Fang J. Comparison of the incidence of perioperative cardiovascular risk events among patients with and without a history of neoadjuvant chemotherapy. Minerva Anestesiol. 2019;85(8):822-9.

18. Chang H, Okwuosa T, Scarabelli T, Moudgil R, Yeh E. Cardiovascular Complications of Cancer Therapy: Best Practices in Diagnosis, Prevention, and Management: Part 2. J Am Coll Cardiol. 2017;70(20):255265.

19. Zeng X, Wang X, Chen H, Liu D, Shi J, Zhang J, Li X, Zhang L, Yang Y, Zhang J: Evaluating the Image Quality of Monoenergetic Images From Dual-Energy Computed Tomography With Low-Concentration and Low-Flow-Rate Contrast Media for the Arterials Supply to the Nipple-Areola Complex in Breast Cancer Compared With Conventional Computed Tomography Angiography. Journal of computer assisted tomography 2020.

20. Eisenberg E, McElhinney P, Commandeur F, Chen X, Cadet S, Goeller M, Razipour A, Gransar H, Cantu S, Miller R, et al. Deep Learning-Based Quantification of Epicardial Adipose Tissue Volume and Attenuation Predicts Major Adverse Cardiovascular Events in Asymptomatic Subjects. Circulation Cardiovascular imaging. 2020;13(2):e009829.

21. Russo R, Di lorio B, Di Lullo L, Russo D. Epicardial adipose tissue: new parameter for cardiovascular risk assessment in high risk populations. J Nephrol. 2018;31(6):847-53.

22. Henson J, Simon T, Kaplan A, Osganian S, Masia R, Corey K. Advanced fibrosis is associated with incident cardiovascular disease in patients with non-alcoholic fatty liver disease. Aliment Pharmacol Ther. 2020;51(7):728-36. 
23. Cai J, Zhang X, Ji Y, Zhang P, She Z, Li H. Nonalcoholic Fatty Liver Disease Pandemic Fuels the Upsurge in Cardiovascular Diseases. Circulation research. 2020;126(5):679-704.

24. Ballestri S, Lonardo A, Bonapace S, Byrne CD, Loria P, Targher G. Risk of cardiovascular, cardiac and arrhythmic complications in patients with non-alcoholic fatty liver disease. World J Gastroenterol. 2014;20(7):1724-45.

25. Younossi Z, Anstee QM, Marietti M, Hardy T, Henry L, Eslam M, George J, Bugianesi E. Global burden of NAFLD and NASH: trends, predictions, risk factors and prevention. Nature reviews Gastroenterology hepatology. 2018;15(1):11-20.

26. Tana C, Ballestri S, Ricci F, Di Vincenzo A, Ticinesi A, Gallina S, Giamberardino MA, Cipollone F, Sutton R, Vettor R, et al: Cardiovascular Risk in Non-Alcoholic Fatty Liver Disease: Mechanisms and Therapeutic Implications. International journal of environmental research and public health 2019, 16(17).

27. Gradishar W, Anderson B, Balassanian R, Blair S, Burstein H, Cyr A, Elias A, Farrar W, Forero A, Giordano S, et al. Breast Cancer, Version 4.2017, NCCN Clinical Practice Guidelines in Oncology. Journal of the National Comprehensive Cancer Network: JNCCN. 2018;16(3):310-20.

28. Kühn J, Meffert P, Heske C, Kromrey M, Schmidt C, Mensel B, Völzke H, Lerch M, Hernando D, Mayerle J, et al. Prevalence of Fatty Liver Disease and Hepatic Iron Overload in a Northeastern German Population by Using Quantitative MR Imaging. Radiology. 2017;284(3):706-16.

29. Marwan M, Koenig S, Schreiber K, Ammon F, Goeller M, Bittner D, Achenbach S, Hell M. Quantification of epicardial adipose tissue by cardiac CT: Influence of acquisition parameters and contrast enhancement. Eur J Radiol. 2019;121:108732.

30. Tang A, Tan J, Sun M, Hamilton G, Bydder M, Wolfson T, Gamst A, Middleton M, Brunt E, Loomba R, et al. Nonalcoholic fatty liver disease: MR imaging of liver proton density fat fraction to assess hepatic steatosis. Radiology. 2013;267(2):422-31.

31. Jayakumar S, Middleton M, Lawitz E, Mantry P, Caldwell S, Arnold H, Mae Diehl A, Ghalib R, Elkhashab M, Abdelmalek $\mathrm{M}$, et al. Longitudinal correlations between MRE, MRI-PDFF, and liver histology in patients with non-alcoholic steatohepatitis: Analysis of data from a phase II trial of selonsertib. Journal of hepatology. 2019;70(1):133-41.

32. F I. F K: Paclitaxel-induced hepatic steatosis in patients with breast cancer. Journal of BUON: official journal of the Balkan Union of Oncology. 2019;24(6):2355-60.

33. Labbe G, Pessayre D, Fromenty B. Drug-induced liver injury through mitochondrial dysfunction: mechanisms and detection during preclinical safety studies. Fundam Clin Pharmacol. 2008;22(4):33553.

34. Hui SCN, So HK, Chan DFY, Wong SKH, Yeung DKW, Ng EKW, Chu WCW. Validation of water-fat MRI and proton MRS in assessment of hepatic fat and the heterogeneous distribution of hepatic fat and iron in subjects with non-alcoholic fatty liver disease. Eur J Radiol. 2018;107:7-13.

35. Gates G, Dore E. Streamline flow in the human portal vein. Journal of nuclear medicine: official publication Society of Nuclear Medicine. 1973;14(2):79-83.

36. Lancellotti P, Anker S, Donal E, Edvardsen T, Popescu B, Farmakis D, Filippatos G, Habib G, Maggioni A, Jerusalem G, et al. EACVI/HFA Cardiac Oncology Toxicity Registry in breast cancer patients: rationale, 
study design, and methodology (EACVI/HFA COT Registry)--EURObservational Research Program of the European Society of Cardiology. Eur Heart J Cardiovasc Imaging. 2015;16(5):466-70.

37. Madonna R, Massaro M, Scoditti E, Pescetelli I, De Caterina R. The epicardial adipose tissue and the coronary arteries: dangerous liaisons. Cardiovascular research. 2019;115(6):1013-25.

38. Packer M. Epicardial Adipose Tissue May Mediate Deleterious Effects of Obesity and Inflammation on the Myocardium. J Am Coll Cardiol. 2018;71(20):2360-72.

39. Christensen R, von Scholten B, Hansen C, Jensen M, Vilsbøll T, Rossing P, Jørgensen P. Epicardial adipose tissue predicts incident cardiovascular disease and mortality in patients with type 2 diabetes.

Cardiovascular diabetology. 2019;18(1):114.

40. Adams L, Anstee Q, Tilg H, Targher G. Non-alcoholic fatty liver disease and its relationship with cardiovascular disease and other extrahepatic diseases. Gut. 2017;66(6):1138-53.

41. van den Berg E, Wolters A, Dullaart R, Moshage H, Zurakowski D, de Meijer V, Blokzijl H. Prescription of statins in suspected non-alcoholic fatty liver disease and high cardiovascular risk, a population-based study. Liver international: official journal of the International Association for the Study of the Liver. 2019;39(7):1343-54.

42. Xia LY, Hu QL, Zhang J, Xu WY, Li XS. Survival outcomes of neoadjuvant versus adjuvant chemotherapy in triple-negative breast cancer: a meta-analysis of 36,480 cases. World J Surg Oncol. 2020;18(1):129.

\section{Figures}

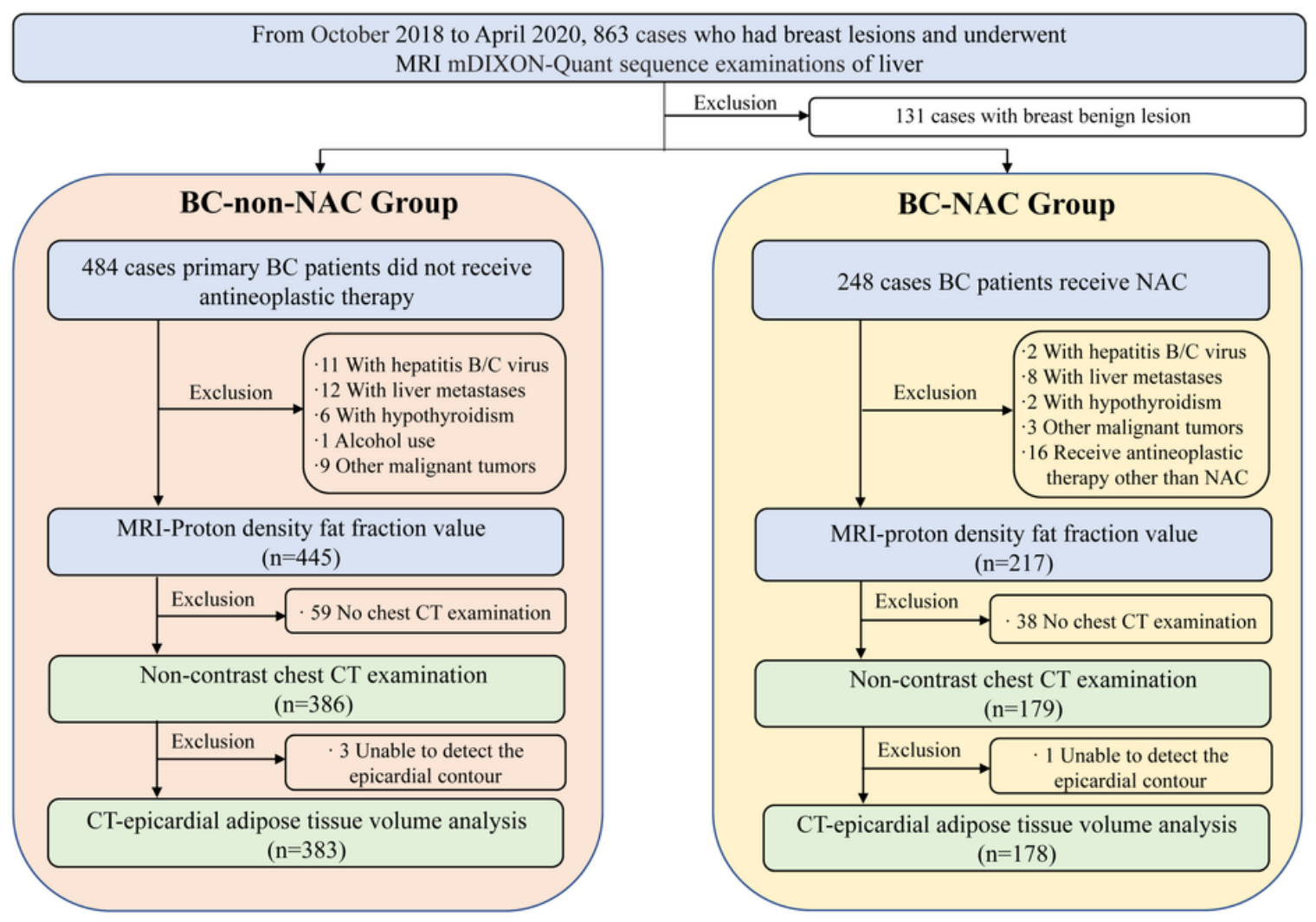

Figure 1 
Flow diagram of participants. $\mathrm{BC}=$ breast cancer; NAC = neoadjuvant chemotherapy.

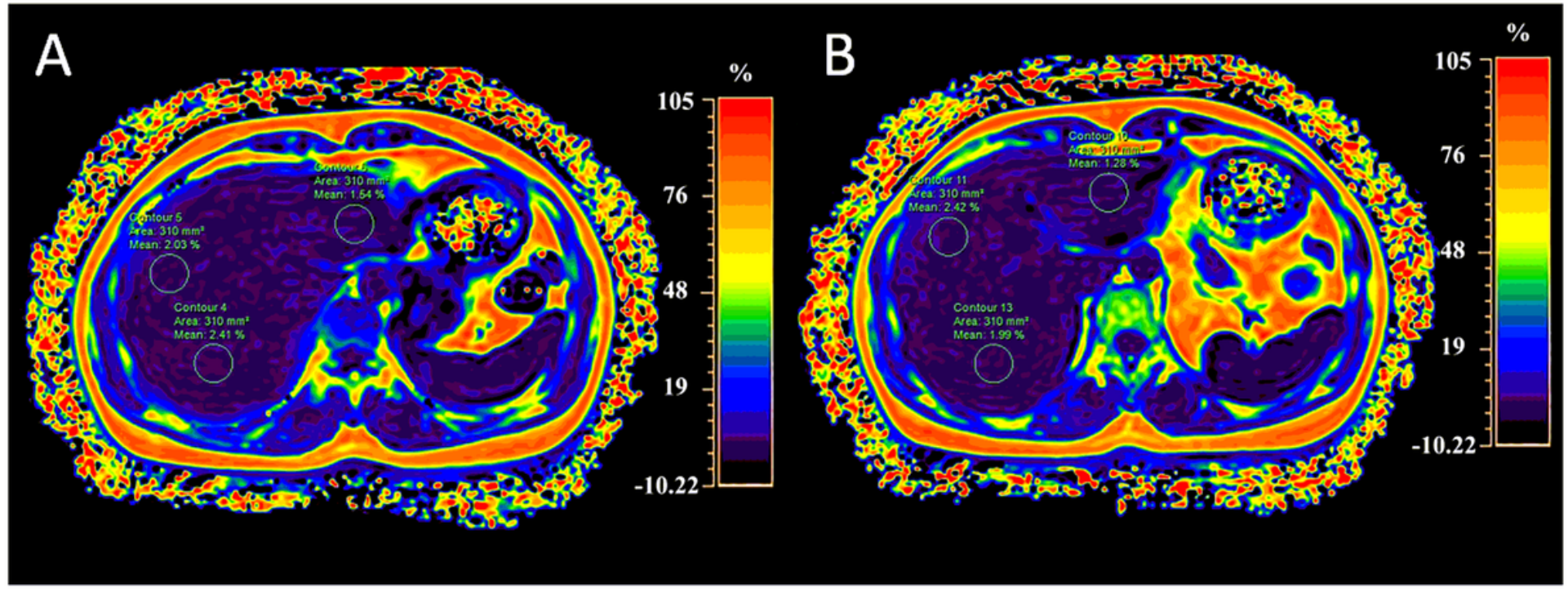

Figure 2

Sample ROIs used to calculate the hepatic PDFF value in transverse sections through the first hepatic portal vein (A) and below the second hepatic portal vein (B). PDFF = proton density fat fraction; ROI = region of interest.
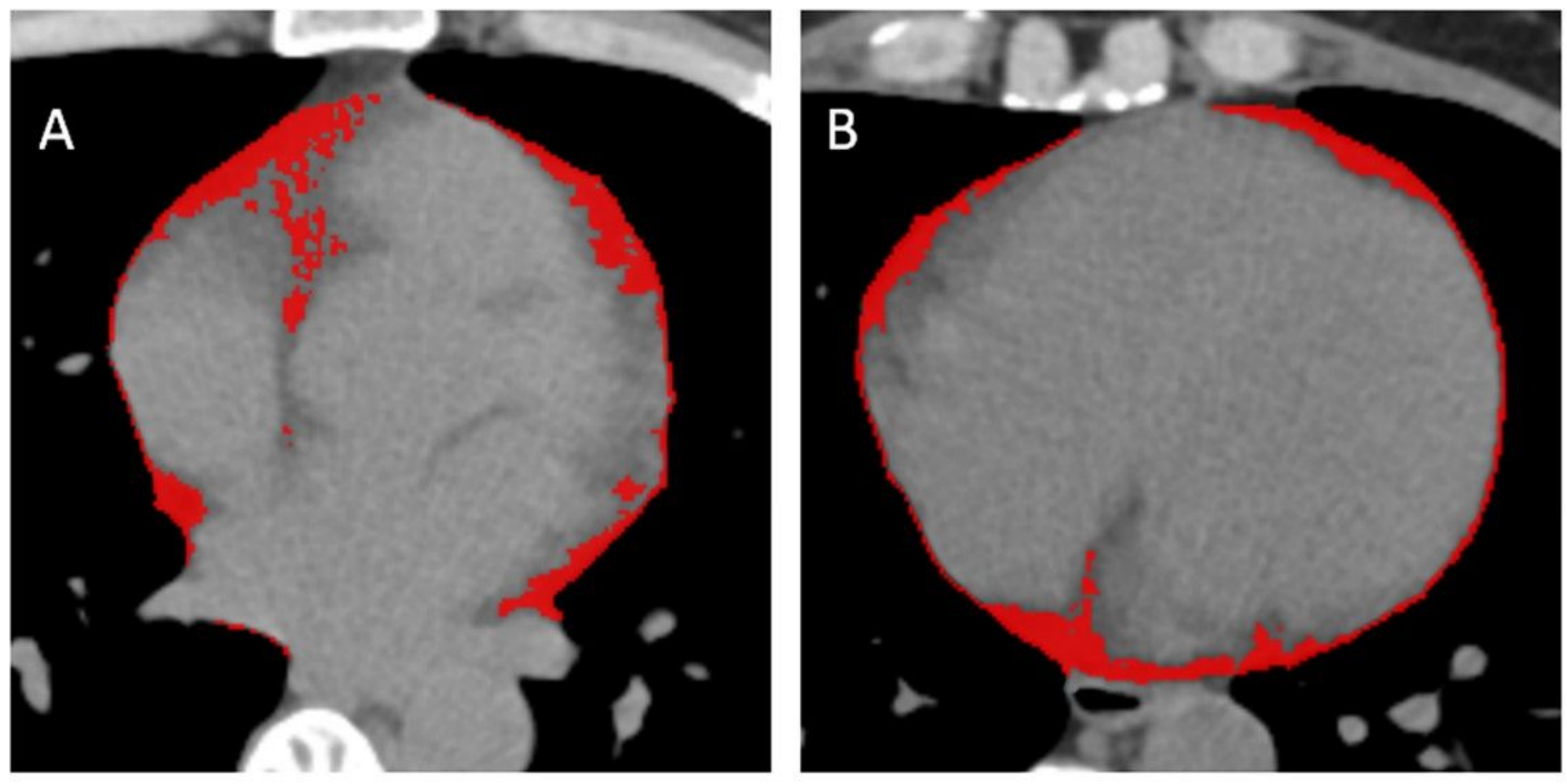

\section{Figure 3}

Semi-automatic quantification of EAT volume on non-contrast image. The contour of the pericardial sac was automatically traced and adjusted by the reader, if necessary. The EAT volume (red) was automatically calculated by the inclusion of all contiguous 3D voxels with CT attenuations between the specified upper threshold (-30 HU) and the lower threshold of -190 HU. EAT = epicardial adipose tissue. 

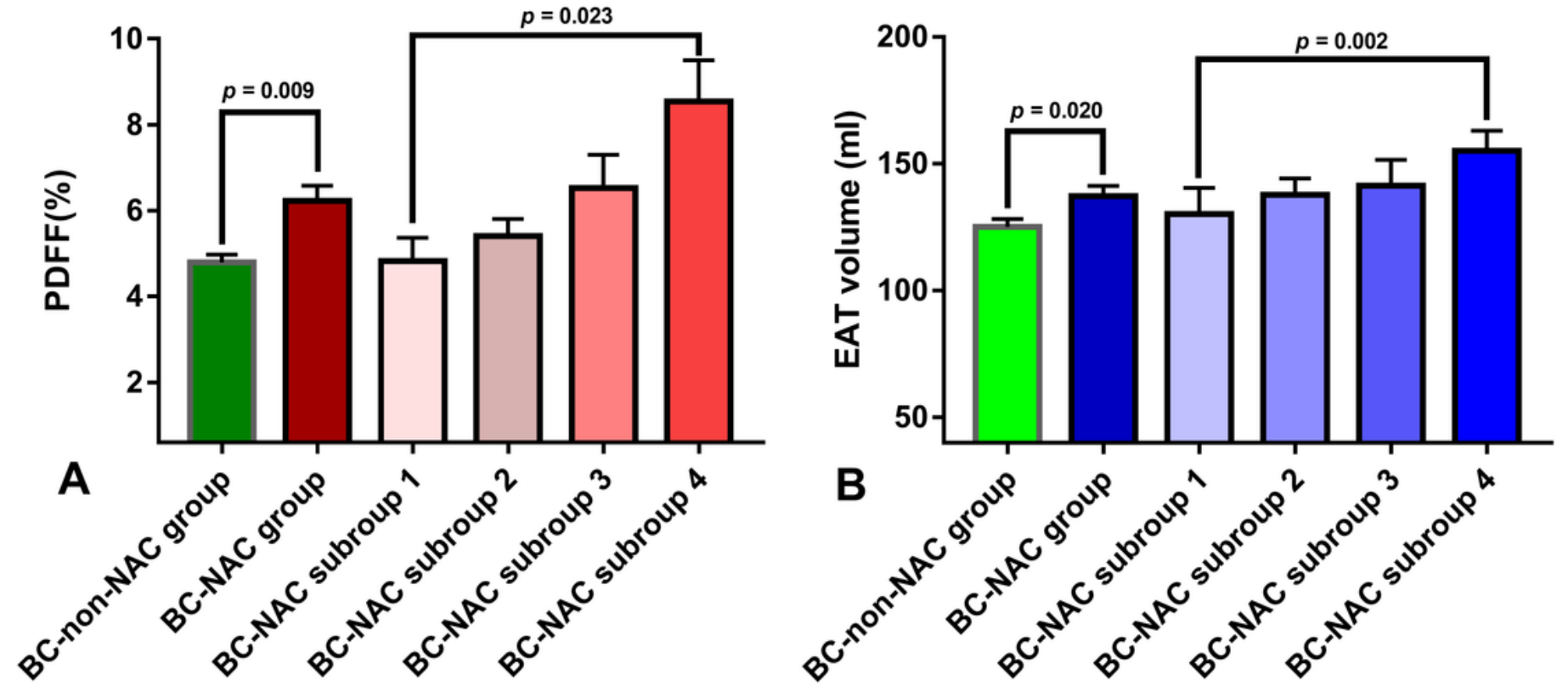

Figure 4

Box plot of the hepatic PDFF value $(A)$ and EAT volume $(B)$ in the BC-non-NAC group, BC-NAC group and BCNAC subgroups. PDFF = proton density fat fraction; $B C=$ breast cancer; NAC = neoadjuvant chemotherapy; EAT = epicardial adipose tissue. 


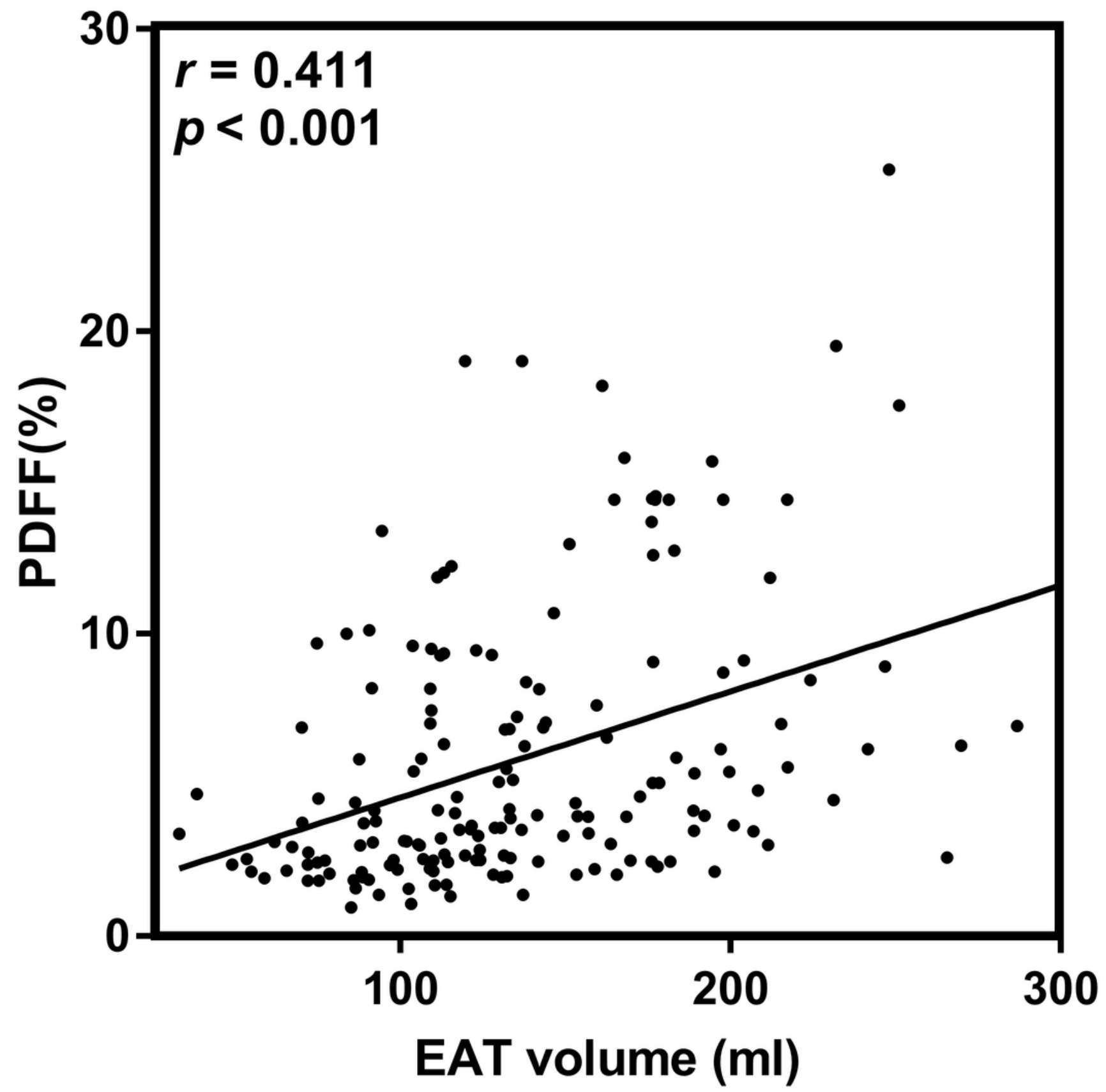

Figure 5

Scatterplots showing MRI proton density fat fraction (PDFF) against the epicardial adipose tissue (EAT). 


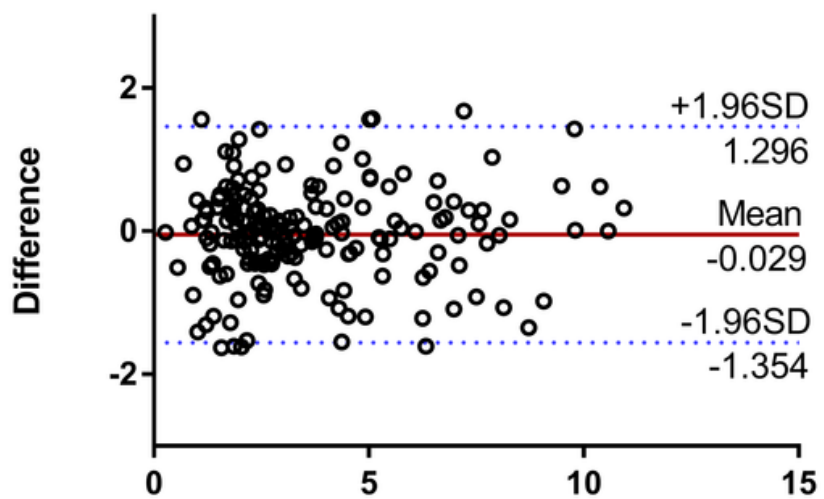

A Mean of PDFF(\%)

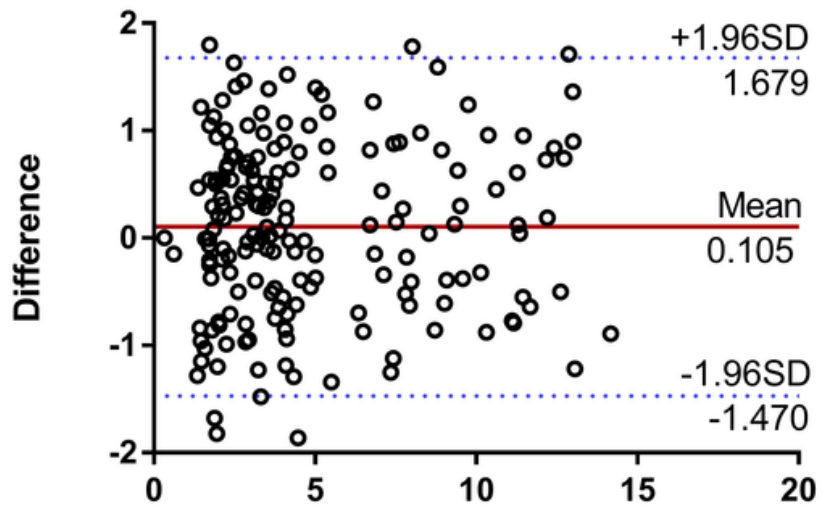

C Mean of PDFF(\%)

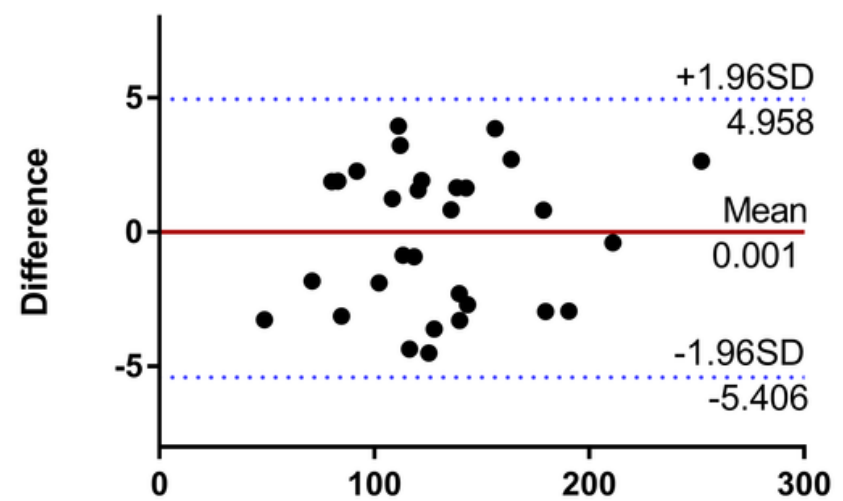

B

Mean of EAT (ml)

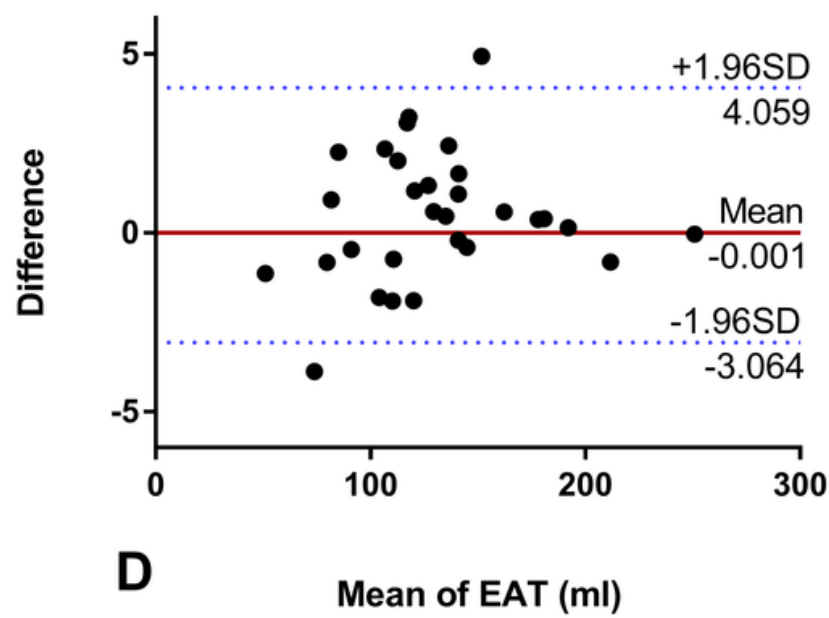

Figure 6

Bland-Altman diagram of PDFF value and EAT volume measured by one observer with an interval of at least 1 month (A-PDFF, B- EAT), and two observers (C-PDFF, D- EAT). PDFF = proton density fat fraction; EAT = epicardial adipose tissue. 